\title{
Comparison of Antibacterial Efficacy Chlorohexidine Gluconate and Saudi Myrrh Mouthwashes in the Oral Cavity
}

\author{
ZAINAH MOHAMAD SAMBAWA ${ }^{1 *}$, FAWZIA HAIF ALKAHTANI', \\ FADILAH SFOUQ ALEANIZY² and FULWAH YAHYA. ALQAHTANI ${ }^{2}$
}

${ }^{1}$ General dentist, Dental Department, Prince Sultan Military Medical City, Riyadh, Saudi Arabia.

${ }^{2}$ King Saud University, College of Pharmacy, Department of Pharmaceutics, Riyadh, Saudi Arabia.

${ }^{*}$ Correspondence author E-mail: zsambawa@ hotmail.com

http://dx.doi.org/10.13005/ojc/320532

(Received: August 05, 2016; Accepted: September 16, 2016)

\begin{abstract}
Myrrh have been traded in the Middle East and North Africa for 5,000 years, Myrrh is an Oleo-gum resin, phytotoxically safe raw material obtained from the stem of various species of the genus Commiphora, in recent years it has been used in pharmaceuticals, food industries. Chlorhexidine gluconate mouthwash is preferably prescribed by dentist as an effective mouthwash. Aim of the study to investigate the antibacterial effect of myrrh aqueous mouthwash (homemade) solution against some selected microbial flora and comparison of the activity with that of commercial chlorohexidine gluconate mouthwash by participation of 9 female volunteers. Antimicrobial activity of Myrrh was investigated and compared with $0.2 \%$ chlorohexidine gluconate mouthwash using agar diffusion method. Escherichia coli 25922, Salmonella 25566, Klebsiella pneumonia 13883, Pseudomonas 27853, Proteus, Staphylococcus aureus 25923, Bacillus, Streptococcus mutans and Candida albicans were used in this study and adjusted according to the Clinical and Laboratory Standards Institute (CLSI). The results revealed that Myrrh produced antimicrobial activity against oral flora and other species of microorganisms which was to some extent comparable with that of Chlorohexidine gluconate. Microbial load detection showed that home-made myrrh mouthwash aqueous solution is an effective as clinically proved mouthwash.
\end{abstract}

keywords: Myrrh, Chlorohexidine, Mouthwash, Antibacterial.

\section{INTRODUCTION}

The oral cavity is comprised of many surfaces, each coated with a bacterial biofilm. Normal oral flora can be considerably changed with poor oral hygiene leading to an increase in their virulence some of these bacteria have been implicated in oral diseases such as caries and periodontitis ${ }^{1}$.

Herbal extracts are globally used as commercial remedies such as peppermint, myrrh, rosemary and miswak which are known as the active 
ingredients in oral preparations ie. tooth gels and mouthwashes.

Myrrh is an oleo-gum-resin obtained from the stem of different species of Commiphora tree ${ }^{2}$. The Commiphora genus (family: Burseraceae) has over 150species distributed around the red Sea in east Africa, Arabia and India ${ }^{3}$.

Investigations have revealed that myrrh contains about 2 to $8 \%$ essential oil (myrrhol), 23 to $40 \%$ resin (myrrhin), 40 to $60 \%$ gum, and 10 to $25 \%$ bitter principles ${ }^{4}$.

In The study by Rao et al. (2001) in mice showed no visible signs of Myrrh toxicity or mortality at oral doses of resin $\leq 3 \mathrm{~g} / \mathrm{kg}$ bodyweight ${ }^{5}$. This was in agreement with Tadesse et al. (2007) who reported that myrrh is phytotoxically safe raw material in industries like pharmaceuticals and food industries ${ }^{6}$. In the field of dentistry minimum inhibitory concentrations of myrrh have been reported to inhibit common bacterial and fungal pathogens, such as Escherichia coli, Staphylococcus aureus, Candida albicans, and Pseudomonas aeruginosa lesion?

Evidence suggested that toothpastes and mouthwashes which contain myrrh are effective in preventing and treating gingivitis ${ }^{8}$. In a recent animal study by Al-Mobeeriek (2011), a diluted myrrh suspension was found to promote the healing and repair of damaged oral tissues ${ }^{9}$.

Trials by Taheri et al. (2011) revealed that myrrh suspension even at low concentration showed healing and repair of damaged tissue when used over a short period of time (less than 2 weeks) however, it can have harmful effects if used in excess or over a long period of time ${ }^{10}$.

Myrrh products are made either in the form of myrrh oil tinctures (diluted with alcohol), or myrrh extracts ${ }^{11}$. So, we have investigated the antibacterial activity of myrrh extract as a homemade mouthwash (colloidal solution) which can be prepared by the patient as an alternative remedy to the synthetic mouthwashes such as Chlorohexidine gluconate. Chlorhexidine gluconate is currently used as an antibacterial mouthwash for reducing plaque and gingivitis.

\section{Aim of the study}

This study was conducted to investigate the antimicrobial activity of Saudi myrrh aqueous mouthwash (homemade) solution against some selected microbial flora in comparison with that of chlorohexidine gluconate mouthwash.

\section{MATERIALS AND METHODS}

Myrrh oleo -resin crystals were purchased from the local market as Saudi myrrh,] ORAXINE Chlorohexidine gluconate mouthwash $0.2 \% \mathrm{w} / \mathrm{v}, 300$ $\mathrm{ml}$ Riyadh pharma. Normal saline 0,9\% w/v Nacl solution, $500 \mathrm{ml}$, pharmaceutical solutions industry, Jeddah.

\section{Preparation of myrrh mouthwash aqueous colloidal solution}

Myrrh oleo-gum resin were purchased from the local market, identified as reddish-brown in Color and complied for its morphology with that described in the USP- $2007^{12}$.

Chemical identification by reaction with nitric acid was positive with purplish violet color Produced instantly?

The myrrh crystals having less impurity were selected, subjected to grinding in a glass mortar and passed through sieve with a mesh size of $224 \mu \mathrm{m}$ Chowdary et al. (2006) ${ }^{13} 0.5$ gram (g) and 1 $\mathrm{g}$ of the collected powder were dissolved in purified sterile distilled warm water $(100 \mathrm{ml})$ and vigorously shacked using magnetic stirrer, Two solutions were obtained having concentrations of $0.5 \mathrm{~g} \% \mathrm{w} / \mathrm{v}$ and $1 \mathrm{~g} \% \mathrm{w} / \mathrm{v}$ with a $\mathrm{pH}$ of 6.6 which is compatible with that of the saliva.

The solutions showed no impurities when seen against light, but it was colloidal and needs to be shaken well before each use.

In literature some herbs were shown to be extracted by sterile distilled water ${ }^{14}$, this method was similar to our work

\section{Microbial preparations}

Escherichia coli 25922, Salmonella 25566, Klebsiella pneumonia 13883, Pseudomonas 27853, Proteus, Staphylococcus aureus 25923, Bacillus, 
Streptococcus pneumoniae and Candida albicans were used in this study. The bacterial suspension was prepared and adjusted by comparison against $0.5 \mathrm{Mc}$-Farland turbidity standard ( $5 \times 10^{7}$ organisms/ $\mathrm{ml}$ ) tubes in accordance with the Clinical and Laboratory Standards Institute (CLSI). The microbes were enriched on Milar Hilton agar Finegoldand Sweeny. (1961) to perform the antimicrobial assay.

\section{Procedure for handling the mouthwash Preliminary test}

One group received one bottle of $0,5 \mathrm{~g} \%$ w/v myrrh solution no 1 and were instructed to rinse the mouth with $5 \mathrm{ml}$ twice daily for 60 seconds and were asked to shake the bottle well before use and to comment on the taste, potency and whether it has an astringent effect on the gum. The same group received $1 \mathrm{~g} \% \mathrm{w} / \mathrm{v}$ myrrh solution no 2 the day after and asked for the same steps. The participants agreed that both solutions had bitter taste, solution no 2 was more potent than solution no 1 and also it showed more astringent effect on the gum when compared to solution no 1. Therefore it was decided to select $1 \mathrm{~g} \% \mathrm{w} / \mathrm{v}$ myrrh solution as the test solution. Since this is a homemade solution, thus, its free from any flavoring or sweeting agents. The participants were informed that myrrh mouthwash was homemade solution therefor, it's free from any coloring, flavoring and sweetening agent.
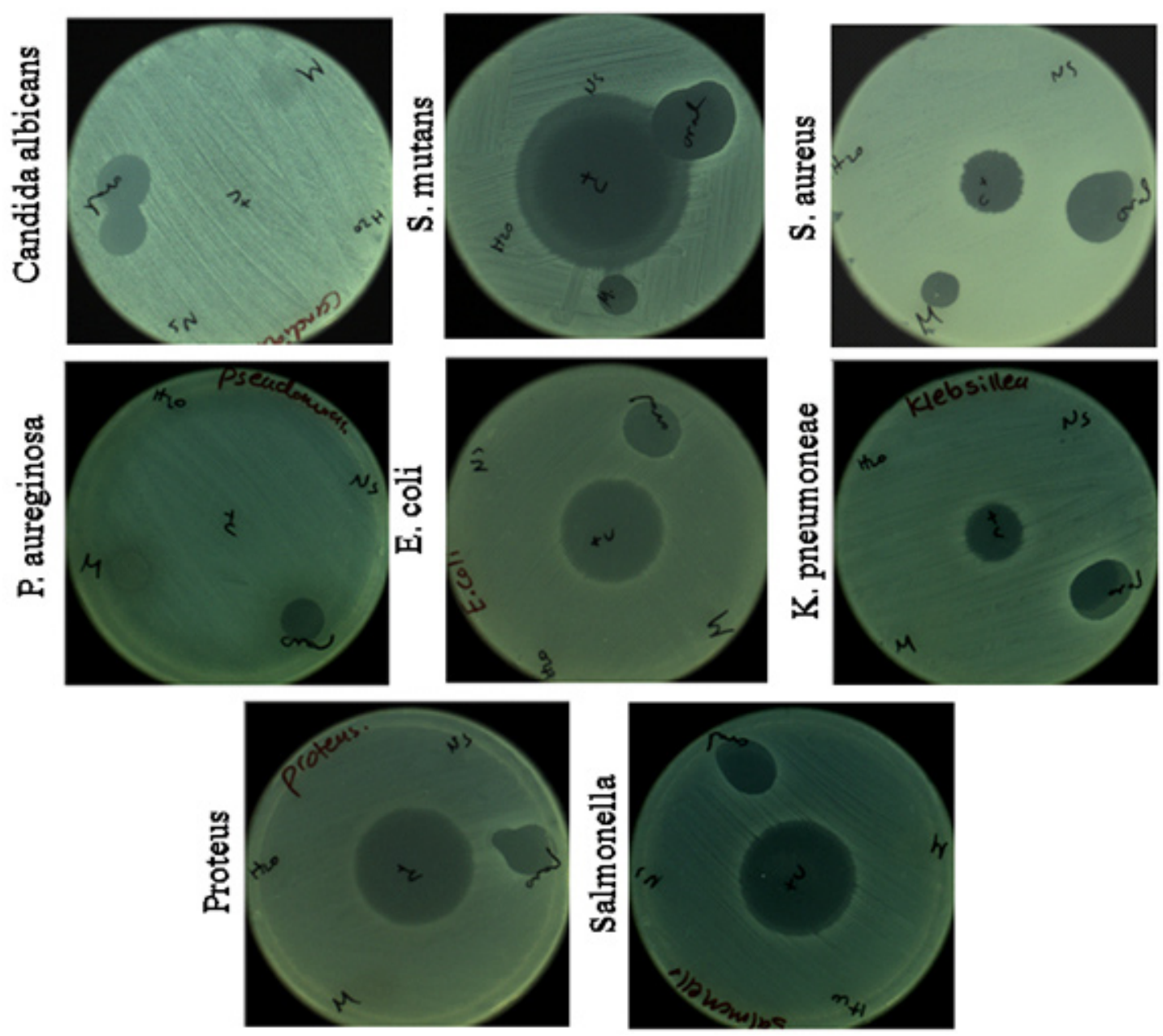

Fig. 1: antimicrobial activity of Myrrh and Chlorohexidine mouthwash solutions against different microorganisms 
Table 1: Shows the effect of mouthwashes in the oral cavity

\begin{tabular}{lcc} 
OD600 & before & after \\
\hline Control 1 & 0.87 & 0.83 \\
Control 2 & 0.83 & 0.78 \\
Control 3 & 0.9 & 0.86 \\
CHX 1 & 0.75 & 0.34 \\
CHX 2 & 0.94 & 0.37 \\
CHX 3 & 0.82 & 0.22 \\
Myrrh 1 & 0.87 & 0.47 \\
Myrrh 2 & 0.94 & 0.48 \\
Myrrh 3 & 0.87 & 0.39 \\
\hline
\end{tabular}

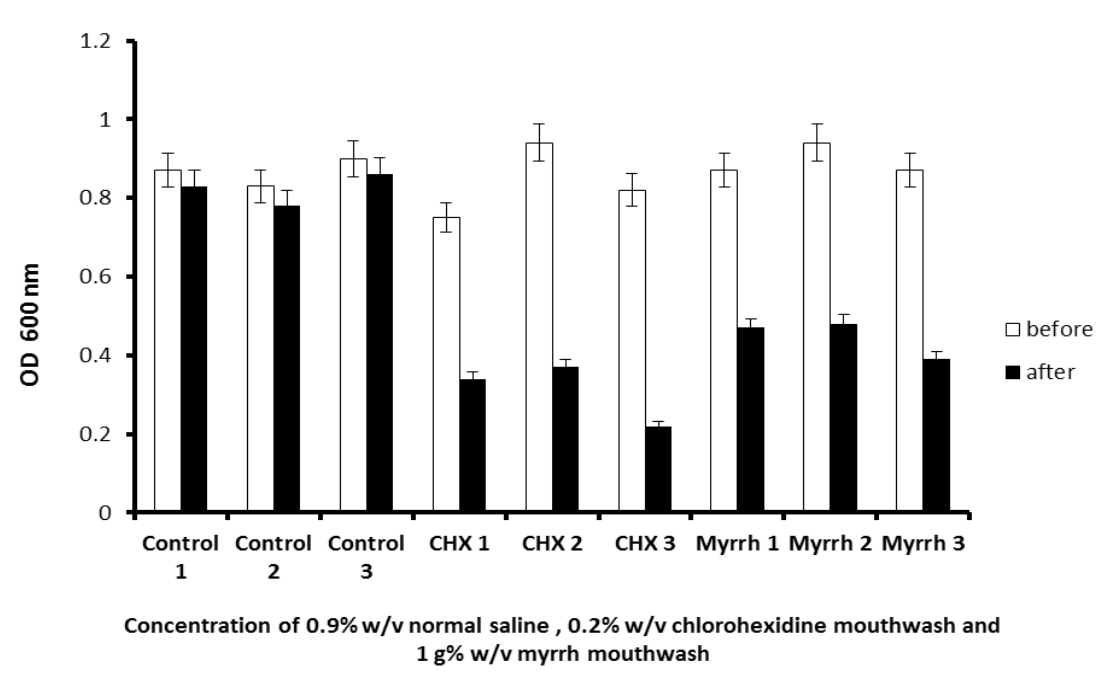

Fig.2 :

\section{Experimental design}

All selected subjects invited to participate in this study were healthy female with good oral hygiene. Microbial load was detected by measuring the OD600 using spectrophotometer for all participants before and after the usage of the mouthwashes. Subjects were randomly divided into 3 groups of 3 s: Group A: 3 participants were asked to rinse for 60 s with chlorhexidine twice a day for one week. Group B: 3 participants were asked to rinse in the same protocol with myrrh mouthwash $1 \mathrm{~g} \% \mathrm{w} / \mathrm{v}$. Group C: 3 participants were asked to rinse with normal saline as a control group.

\section{Antimicrobial Assay}

$5 \mathrm{mg}$ of Saudi myrrh was dissolved in $500 \mathrm{ml}$ normal saline (NS). Using the agar diffusion method, was adapted from Mahajan et al, $(2016)^{14}$, antimicrobial activity of myrrh against Gram-negative strains (Escherichia coli 25922, Salmonella 25566, Klebsiella pneumonia 13883, Pseudomonas 27853, and Proteus), Gram-positive strains (Staphylococcus aureus 25923, Bacillus, and Streptococcus pneumoniae) and Fungi (Candida albicans). Inhibitory activity was determined by the agar-diffusion method in comparison with Ampicillin in accordance with the Clinical and Laboratory Standards Institute (CLSI). An amount of $20 \mu \mathrm{l}$ of Myrrh solution were applied into the culture medium. After $24 \mathrm{~h}$ at $37{ }^{\circ} \mathrm{C}$, inhibition zone diameters were measured by subtracting the well diameter. $0.12 \%$ chlorhexidine and normal saline were used as positive and negative controls, respectively and subjected to the same procedures applied to the tested extracts. 


\section{RESULTS AND DISCUSSION}

In the present study Saudi myrrh mouthwash $1 \mathrm{~g} \% \mathrm{w} / \mathrm{v}$ aqueous colloidal solution and $0.2 \%$ Chlorhexidine mouthwash was used .The antibacterial effects of the mouthwashes were compared on oral microbial flora and other microorganisms.

The microorganisms tested in the samples were mainly Escherichia coli 25922, Salmonella 25566, Klebsiella pneumonia 13883, Pseudomonas 27853, Proteus, Staphylococcus aureus 25923, Streptococcus pneumonia, streptococcus mutans and Candida albicans.

The results of antimicrobial assay Shown in Figure 1 revealed that Myrrh produced antimicrobial activity against oral flora (Staphylococcus aureus, streptococcus mutans and Candida albicans).and other microorganisms which was to some extent comparable to Chlorohexidine gluconate.

The results of the microbial load detection shown in Figure 2. Illustrates the comparable antimicrobial activities of chlorhexidine mouthwash and Myrrh mouthwash after using a control group of Normal saline.

\section{DISCUSSION}

In a recent study done by Almekhlafi et al. (2014) ${ }^{11}$ myrrh was used as an alcoholic mouthwash solution of a $200 \mathrm{ig} / \mathrm{ml}$ concentration( $2 \mathrm{~g} \% \mathrm{w} / \mathrm{v}$ ) which had exhibited higher antibacterial efficacy than chlorohexidine under test using microbial oral flora (Staphylococcus aureus, candida albicans and Strep. Mutans) .whilst the concentration of myrrh under test in our work is half of that, although it was comparable with chlorohexidine gluconate using similar microbial flora.In order to optimize the antibacterial activity of our myrrh solution it was suggested to increase the concentration to prepare $1.5 \mathrm{~g} \% \mathrm{w} / \mathrm{v}$ aqueous solution, but the solubility showed difficulties in obtaining the colloidal solution, unless solubilizing and stabilizing agents should be added .
The antimicrobial effect of $0.2 \%$ chlorohexidine mouthwash was also reported by some workers as follows: Kaim JM et al $(1989)^{15}$, Neeti Bajaj et al.( 2011) ${ }^{16}$, Almekhlafi et al (2014) and Mahajan et al( 2016) ${ }^{14}$ who had confirmed its Medical uses for the treatment of oral diseases,this is in agreement with our results which showed an antibacterial effect of the commercial products against oral flora under test.

For the future study masking the bitter taste using artificial sweetening agents such as (splenda:dextrose maltodextrin sucralose) by Mcneil Nutritionals ,LC Fort Washington or using flavoring agents such as peppermint extract to be added according to patients desire. In addition the sample size of participants to be increased using the same bacterial flora .

\section{CONCLUSION}

It is suggested that myrrh $1 \mathrm{~g} \% \mathrm{w} / \mathrm{v}$ aqueous solution can be used as an effective homemade mouthwash as an alternative to (chlorohexidine gluconate) mouthwash because it could be easily prepared by the patient besides its coast effective.

In dentistry, Myrrh extracts may be an alternative remedies for commercially synthetically made mouthwash such as chlorohexidine gluconate for oral health care, because the latter one will mainly produce staining of teeth and tongue surface. In addition there will be loss of taste perception and other several adverse reactions ${ }^{17}$.

\section{ACKNOWLEDGMENTS}

We express our gratitude to both professors, Dr. Omaima ALGohary Prof. of pharmaceutics, college of pharmacy and Dr. Sulaiman Alharbi Prof. of Botany \& Microbiology, College of Science, King Saud University for their advice and guidance and to the research Centre of the Science and Medical studies Departments at King Saud University for financial support. The authors would like to thank all participants in this study. 


\section{REFERENCES}

1. Albandar, J. M., J. A. Brunelle, and A. Kingman. 1999. Destructive periodontal disease in adults 30 years of age and older in the United States, J. Periodontol, 1988-1994.. 70:13-29.

2. El Ashry E., Rashed N., Salma O., Saleh A. Components, therapeutic value and uses of myrrh, Pharmazie, 2003, 58(3),163-168.

3. Ali Awadth N., Wurster M., Lindequist U. Joeobp,2009, 12(2),244-249.

4. Hanus LO, Rezanka T, Dembitsky VM, Moussaieff A. Myrrh-Commiphora chemistry. Biomed Pap Med Fac Univ Palacky Olomouc Czech Repub 2005; 149: 3-27.

5. Rao RM, Khan ZA, Shah AH. Toxicity studies in mice of Commiphora molmol oleogumresin. J Ethnopharmacol; 2001, 76: 151 154.

6. Tadesse W, Desalegn G, Alial R, Natural gum and resin bearing species of Ethiopia. Invest Agrar: Sist Recur For; 2007. 16: 211 221.

7. Dolara P, Corte B, Ghelardini C, Pugliese AM, Cerbai E,Menichetti S, Lo Nostro A. Local anaesthetic, antibacterial and antifungal properties of sesquiterpenes from myrrh. Planta Med 2000; 66: 356-358.

8. Saeidi M, Azadbakht M, Semnani K, Khandan M. Formulation of herbal toothpaste from chamomile and myrrh, a preliminary clinical evaluation on bleeding gum. J Mazandaran Univ Med Sci 2003; 13: 61-69.

9. Al-Mobeeriek A. Effects of myrrh on intra-oral mucosal wounds compared with tetracyclineand chlorhexidinebased mouthwashes. Clin Cosmet Investig Dent 2011; 3: 53-58.
10. Taheri JB; Azimi S; Rafieian N; Zanjani HA, Herbs in dentistry. International Dental Journal. 2011, 61(6):287-96.

11. Sadik Almekhlafi, Anes A. M. Thabit, Ameen M. I. Alwossabi, Nasser Awadth, Abdulbaqi A. M. Thabet and Zaid Algaadari, Antimicrobial activity of Yemeni myrrh mouthwash, Journal of Chemical and Pharmaceutical Research, 2014, 6(5):1006-1013.

12. USP, the .United states pharmacopieal convension, 30th Edition, USA, 2007, 26912692.

13. Chowdary K, Mohapatra P, Krishna MN ,Evaluation of olibanum and its resin as rate controlling matrix for controlled release of diclofenac. Indian J Pharm Sci; 2006, 68: 497 - 500 .

14. Mahajan, Rupali.; Khinda, Paramjit. Kaur.; Amarjit, S. G.; Jyotinder, K.; Saravanan, S. P.; Akhilesh, S.; Meenu, T.; Vaibhav, J. European Journal of Medicinal Plants. 2016, 13, 1-11

15. Kaim JM, Gultz J, Do L, Scherer W. An in vitro investigation of the antimicrobial activity of an herbal mouthrinse. J Clin Dent. 1998;9:4648.

16. Neeti Bajaj and Shobha Tandon ${ }^{1}$ The effect of Triphala and Chlorhexidine mouthwash on dental plaque, gingival inflammation, and microbial growth, Int $J$ Ayurveda Res. 2011 Jan-Mar; 2(1): 29-36

17. Hepso HU,Bjornland T, Skoglund LA, side-effects and patient acceptance of $0.2 \%$ versus $0.1 \%$ chlorohexidine usedas a post-operative prophylactic mouthwash. Int J Oral Maxillofacial Surgery 1988., 17:17-20. 